\title{
A Hot-Electron Direct Detector for Radioastronomy
}

\author{
Boris S. Karasik", William R. McGrath, and Henry G. LeDuc \\ Jet Propulsion Laboratory, Califorria Institute of Technology, Pasadena, CA 91109 \\ Michael E. Gershenson \\ Dept. of Physics \& Astronomy, Rutgers University, Piscataway, NJ 08854
}

\begin{abstract}
A hot-electron transition-edge superconducting bolometer with adjustable thermal relaxation speed is proposed. The bolometer contacts are made from a superconductor with high critical temperature which blocks the thermal diffusion of hot carriers - into the contacts. Thus electron-phonon interaction is the only mechanism for heat removal. The speed of thermal relaxation for hot electrons in a nanometer-size superconducting bolometer with $T_{c}=100-300 \mathrm{mK}$ is controlled by the elastic electron mean free path $l$. The relaxation rate behaves as $T^{\prime} l$ at subkelvin temperatures and can be reduced by a factor of 10 100 by decreasing $l$. Then an antenna- or waveguide-coupled bolometer with a time constant $\sim 10^{-3}$ to $10^{-5} \mathrm{~s}$ will exhibit photon-noise limited performance at millimeter and submillimeter wavelengths. The bolometer will have a figure-ofmerit NEP $\sqrt{ } \tau=10^{-22}-10^{-21} \mathrm{~W} / \sqrt{\mathrm{Hz}}$ at $100 \mathrm{mK}$ which is $10^{3}$ to $10^{4}$ times better (ie: smaller) than that of a state-of-the-art bolometer. A tremendous increase in speed and sensitivity will have a significant impact for observational mapping applications.
\end{abstract}

\section{INTRODUCTION}

Future submillimeter radioastronomy missions will require significant improvement of the sensitivity of radiation detectors. Use of the space interferometers with cooled telescopes will reduce the background-limited noise-equivalent power (NEP) down to $10^{-19} \mathrm{~W} / \mathrm{VHz}$ [1]. Better detectors will be needed to take advantage of these new telescopes. Bolometers are most the promising candidates to meet these future needs. Currently, state-of-the-art bolometers offer the NEP of the order of $10^{-17} \mathrm{~W} / \sqrt{\mathrm{Hz}}$ at $0.1 \mathrm{~K}$ along with a $-1 \mathrm{~ms}$ time constant [2]. The high sensitivity of these bolometers is achieved using carefully-engineered mechanical suspensions which provide high thermal isolation of the absorber and the thermometer from the heat sink. Specially-designed spiderweb suspensions also play the role of radiation absorber at the same time. For even higher sensitivity, the thermal conductance between the device and the heat sink should be reduced. In general, this slows down the bolometer response which, in turn, increases the $1 /$ f-noise contribution and limits the rate of data flow from the instrument.

Manuscript received April 30, 1999.

This research was performed by the Center for Space Microelectronics Technology, Jet Propulsion Laboratory. Califomia Institute of Technology, and was sponsored by NASA, Office of Space Science.

"Email: Boris.Karasik@jpl.nasa.gov

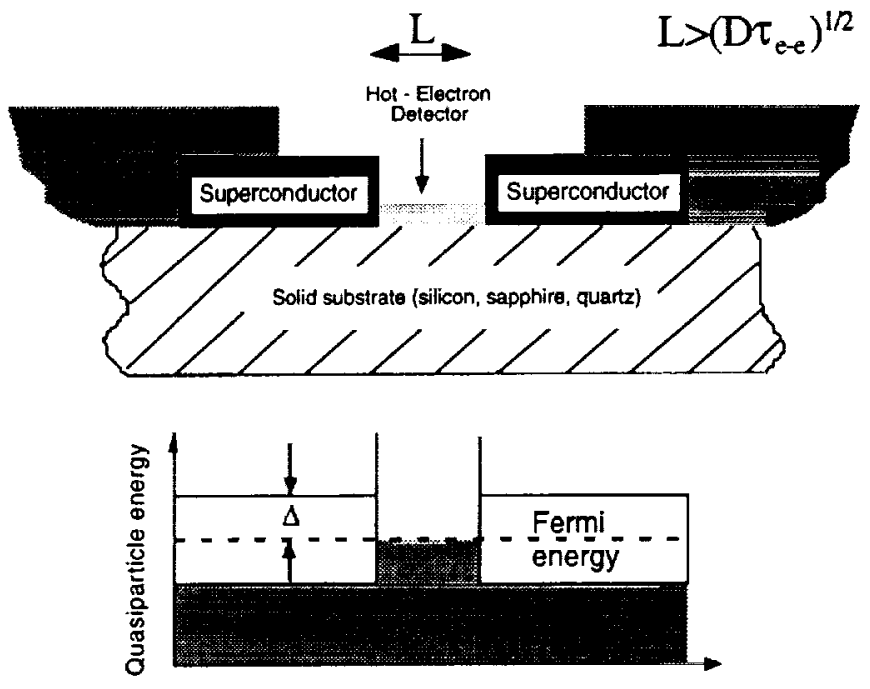

Fig. 1. Schematic diagram of the detector design. The TES is surrounded by superconducting "Andreev mirrors" which prevent the leakage of the thermal energy into the contacts. The energy gap in the TES is suppressed while in the contacts it is fully open. The size of superconducting contacts is small to avoid undesirable if loss at high frequencies.

We propose a new approach for improving the sensitivity of bolometers for use at millimeter to far-infrared wavelengths. The innovative idea is to use an antenna-coupled hot-electron transition-edge sensor (TES) with impuritycontrolled electron-phonon relaxation time. The small heat capacity of a micron or even submicron size microbridge, high thermal resistance of superconducting leads, and finally, properly adjusted electron-phonon relaxation time at ultra-low temperatures provides the high sensitivity of this novel detector. It is noteworthy that the electron-phonon relaxation time is sensitive to the inelastic electron scattering by vibrating impurities and to the modification of the phonon spectrum in thin films. This allows one to regulate the characteristic time constant and to avoid losses of sensitivity because of the excessively large bolometer speed. These detectors will have a figure-of-merit, $N E P \sqrt{ } \tau$, up to four orders of magnitude better (ie: smaller) than that for conventional bolometers. We expect that an antenna- or waveguide-coupled bolometer with a time constant $\tau \sim 10^{-3}$. $10^{-4} \mathrm{~s}$ at $T=0.1-0.3 \mathrm{~K}$ will exhibit photon-noise limited performance in millimeter, submillimeter and infrared wavelengths. Alternatively, the proposed bolometer could operate at higher temperatures with still superior sensitivity. 


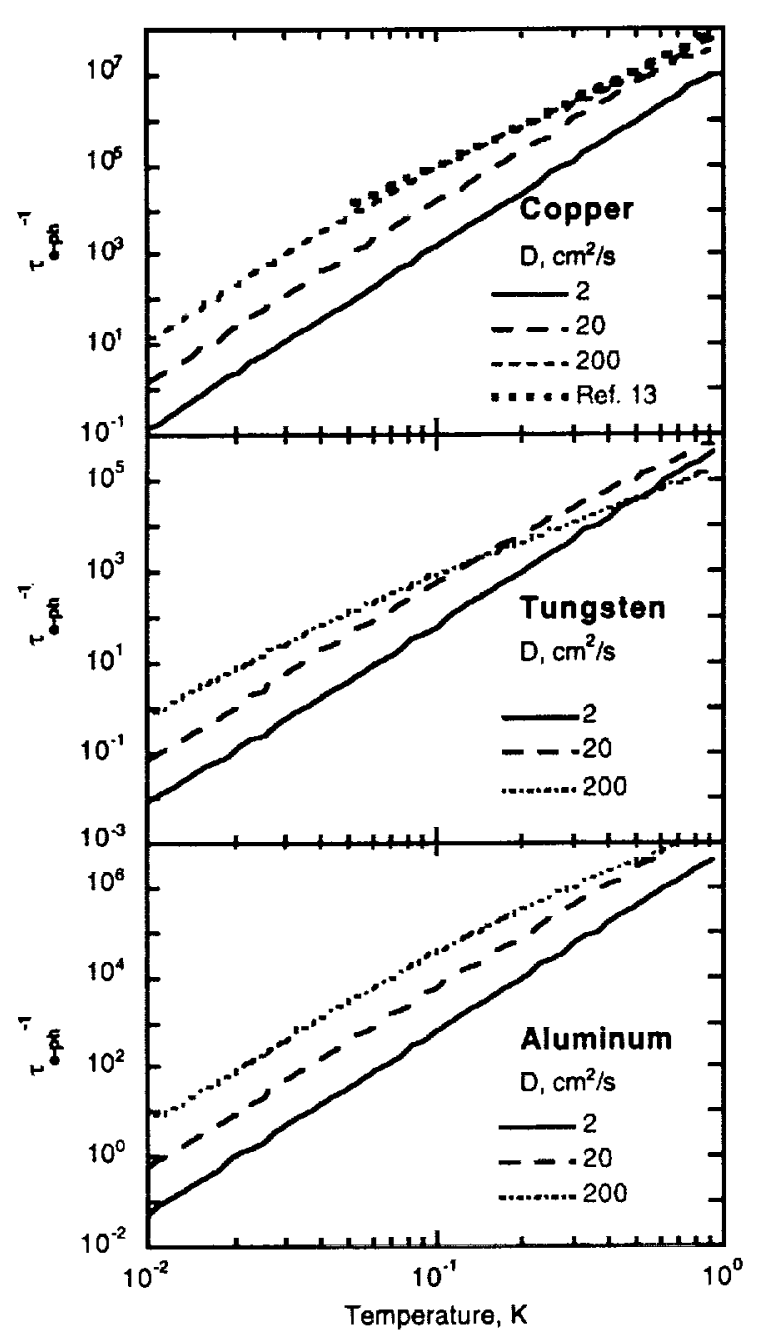

Fig 2. The electron-phonon relaxation rate, $\tau_{\text {e.ph }}{ }^{-1}$, in some metals. The experimental data for $C u$ were obtained for the diffusivity $D=200 \mathrm{~cm}^{2} / \mathrm{s}$.

This device can significantly increase the science return and reduce the cost of the future radioastronomy missions.

\section{Device Concept}

The hot-electron bolometer may be viewed as the limiting case of a conventional bolometer when the thermal conductance due to the electron-phonon coupling is much smaller than those for the phonon escape from the film to the substrate and for the heat transfer between the substrate and the environment. At subkelvin temperatures this is especially easily to achieve if the device is fabricaled on a highlythermal-conductive bulk substrate. For 10-30 nm thick films, the phonon escape time is $<1 \mathrm{~ns}$ and the electron-phonon relaxation is the slowest process which governs the energy relaxation.

A practical implementation of the hot-electron mechanism at subkelvin temperatures requires special design of the contact areas (Fig. 1). The leads should be made from a superconductor with a high critical temperature than the bolometer material. A large superconducting gap, $\Delta$, creates a

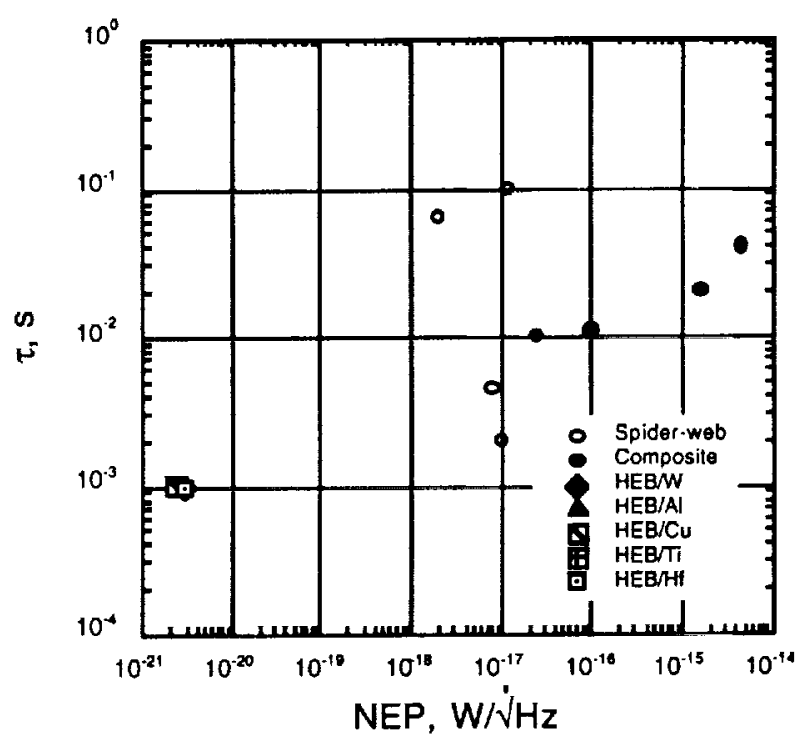

Fig. 3. Comparison of the performance of the state-of-the-art bolometers (circles) and that for HEB detectors of $0.5 \times 0.2 \times 0.01 \mu \mathrm{m}^{3}$ volume. It is assumed that the time constant for HEBs can be adjusted to $1 \mathrm{~ms}$ at $0.1 \mathrm{~K}$. The NEP data for HEB fall very close to each other so the symbols overlap.

barrier for the energy leakage of the electrons energy from the detector. The bias and if currents will flow freely through the structure, whereas the outdiffusion of hot electrons with energies $\varepsilon<\Delta$ will be blocked by the Andreev reflection. A similar design for the contacts was proposed for a normalinsulator-superconductor (NIS) HEB sensor [3]. We prefer to use a superconducting TES because of its higher responsivity, and lack of undesirable shot-noise effects. The TES plays the role of both absorber and temperature sensor, and in the vicinity of the superconducting transition, the bolometer is in a mixed state and represents a simple resistive load for incoming radiation.

As in the case of a conventional bolometer, the sensitivity limit of a HEB detector is given by thermodynamic fluctuations and the corresponding NEP is:

$$
N E P=\sqrt{4 k_{B} T^{2} G_{e-p h}}=\sqrt{4 k_{B} T^{3} W / \tau_{e-p h}} .
$$

where $G_{\text {e-ph }}=C / \tau_{\text {e-ph }}$ is the effective thermal conductance for the heat transfer between electrons and phonons; and $C_{e}=\gamma T V$ is the electron heat capacity, $\gamma$ is the Sommerfeld constant, $V$ is the film volume. In contrast to the case of conventional bolometers, where the thermal conductance depends on the mechanical design of the bolometer suspension, the NEP of an HEB depends on volume.

It is common to characterize the performance of bolometers by the figure-of-merit $N E P \sqrt{ } t$, that is, the minimum energy that can be detected by a bolometer. In the case of hot-electron detectors, this figure depends only on the specific heat of electrons, whereas for conventional bolometers there is an additional large contribution due to the specific heat of phonons in the whole device (the absorber, 
the sensing element, and the thermometer). Thus, in principle, the hot-electron detectors offer the maximum possible sensitivity among all types of bolometers with the same time constant.

The time constant $\tau$ of the hot-electron detector is determined by the inelastic electron-phonon relaxation time $\tau_{\varepsilon \cdot p h}[4]$ (for a conventional bolometer, $\tau$ is determined by the ratio of the total heat capacity to the design-dependent thermal conductance). At $T=4.2 \mathrm{~K}$, the electron-phonon time can be as small as $10^{-10} \mathrm{~s}$; for this reason, the $N b N$ devices of this type are used as terahertz mixers with the intermediate frequency response up to $3-4 \mathrm{GHz}$ [5]. On the other hand, the time constant $\tau$ required for direct detection applications is typically of the order of $10^{-3} \mathrm{~s}$. Thus, to minimize the NEP, one has to adjust the electron-phonon time $\tau_{e \cdot p h}$ to the required $\tau \sim 10^{-3} \mathrm{~s}$.

The use of a TES instead of a normal metal bolometer with an NIS-junction [3] allows to explore the electro-thermal feedback (ETF) [6]. The NEP of a bolometer is not affected by the ETF [7], therefore for a hot-electron TES, one can attain both sensitivite and fast performance in two steps: first, increasing the intrinsic response time (झincreasing the sensitivity) by adjusting the disorder in the film, and, second, decreasing the response time again employing the ETF.

\section{ELECTRON-PHONON INTERACTION IN DISORDERED METALS}

The electron-phonon interaction in impure metals depends on the material disorder. This fact has not been appreciated by the bolometric community yet, though there is an extensive literature on this subject (see [8,9] for a review). The crucial parameter driving $\tau_{e \cdot p h}$ is $q \ell$ ( $q$ is the phonon wavevector). In very clean metals $(q \ell>1), \tau_{\ell \cdot p h} \sim T^{3}$. However, at subkelvin temperatures, the opposite limit $q \ell<1$ takes place even in clean thin films because of the diffusive scattering of electrons at the film surface. In the latter case, $\tau_{\text {e.ph }} \sim T^{4} l^{l}$ [10]. In the intermediate range $q l \sim 1$, the temperature dependence is weaker and the disorder dependence is less pronounced $[8,11]$. Theory [10] agrees well with the most of experimental data (see [8]). Some predictions of the theory are shown in Fig. 2 (more detailed comparison between the theory and experiments can be found in [8,12]). For instance, $\tau_{e-p h}$ is of the order of $1 \times 10^{-5} \mathrm{~s}$ at $T=0.1 \mathrm{~K}$ in clean $\mathrm{Cu}$ films [13]. By introducing disorder, $\tau_{\text {e-ph }}$ can be easily decreased by two orders of magnitude. Similar effects are expected for the other metals, e.g. W and AI (see Fig. 2), which can be interesting for HEB detectors (see below). The control of the electron mean free path can be achieved by making films thinner or/and by irradiating films with highenergy ions [12]. The theory predictions obtained for $\tau_{r \cdot p h}$ in normal metals, apply to the electron-phonon interaction in the resistive state of a superconducting film due to a large concentration of quasiparticles.

\section{PERFORMANCE DISCUSSION}

Figure 3 shows the performance of HEBs fabricated from superconductors with the critical temperature $T_{c}$ around $0.1 \mathrm{~K}$ $(W, H f)$ as well as for $A l, T i$ and $C u$, which can be used for fabrication of proximity bi-layers with adjustable $T_{\mathrm{c}}$ [14]. Although the parameters of bi-layer structures $\left(\gamma\right.$ and $\left.\tau_{e-p h}\right)$ are not well known, one can expect that the specific heat would be somewhere in between of those for a normal metal and a superconductor. In principle, the materials with smaller $\gamma$ offer better sensitivity (see Eq. 1). In this sense, the best materials are semimetals $(\mathrm{Bi}, \mathrm{Sb})$ offering an NEP as low as $\sim 10^{-22} \mathrm{~W} \sqrt{\mathrm{Hz}}$. However, it is clear that even for conventional materials, the controllable adjustment of the electron-phonon time to the required time constant will allow to reach the $N E P$ by three to four orders of magnitude lower than the NEP of existing ultra-sensitive conventional bolometers.

\section{CONCLUSION}

We have proposed a new type of direct detector based on a hot-electron TES where the electron-phonon relaxation time is adjusted by proper control of the film disorder. It results in a very low NEP which will allow for photon-noise limited performance of the detector from millimeter to infrared wavelengths. A number of superconducting materials are potentially suitable for fabrication of the TES. Tests of new devices will begin soon.

\section{ACKNOWLEDGMENT}

Fruitful discussions with A. Kozhevnikov L. Kuzmin, K. Likharev, D. Prober, R. Schoelkopf, and A. Sergeev are greatly appreciated.

\section{REFERENCES}

[I] J.C. Mather, S.H. Moseley, Jr., D. Leisawitz, E. Dwek, P. Hacking, M. Harwit, L.G. Mundy, R.F. Mushotzky, D. Neufeld, D. Spergel, and E.L. Wright, http://xxx.lanl.gov/abs/astro-ph/9812454

[2] J.J. Bock, J. Glenn, S.M. Grannan, K.D. Irwin, A.E. Lange, H.G. LeDuc, and A.D. Turner, SPIE 3357, 297 (1998).

[3] M. Nahum and J.M. Martinis, Appl. Phys. Lett. 63, 3075 (1993); A. Vystavkin, D. Chouvaev, L. Kuzmin, M. Tarasov, E. Anderstedt, M. Willander, and T. Claeson, SPIE 3465, 44 I (1998).

[4] E.M. Gershenson, M.E. Gershenzon, G.N. Gol'tsman, A.D. Semenov, and A.V. Sergeev, Sov. Phys.-JETP Lett. 36, 297 (1982); Sov.Phys.-JETP 59, 442 (1984); Solid Stare Commun. 50, 207 (1984).

[5] S. Svechnikov, G. Gol'tsman, B. Voronov, P. Yagoubov, S. Cherednichenko, E. Gershenzon, V. Belitsky. H. Ekström, E. Kollberg, A. Semenov, Yu. Gousev, and K. Renk. IEEE Trans. Appl. Supercond. 7. 3395 (1997)

[6] A.T. Lee, P.L. Richards, S.W. Nam, B. Cabrera, and K.D. Irwin, Appl. Phys. Lett. 69, 1801 (1996).

[7] W.A. Mels, M.P. Bruijn, H.F.C. Hoevers, M. Frericks, F.B. Kiewiet. A.C. Bento, and P.A.J. de Korte, Proc. SPIE 3445, 255 (1998).

[8] N.G. Ptitsina. G.M. Chulkova, K.S. Il'in, A.V. Sergeev, F.S. Pochinkov, E.M Gershenzon, and M.E. Gershenzon, Phys. Rev. B 56, 10089 (1997).

[9] B.I. Betevtsev, Yu.F. Komnik, and E.Yu. Beliayev, Phys. Rev. B 58, 8079 (1998)

[10] M.Yu. Reizer and A.V. Sergeev, Sov. Phys. - JETP 63, 616 (1986)

[II] B.S. Karasik and A.V. Sergeev, unpublished.

[12] K.S. Il'in, N.G. Ptitsina, G.N. Gol'tsman, E.M. Gershenzon, B.S. Karasik. E.V. Pechen and S.I. Krasnosvobodtsev, Phys. Rev. B 57. 23 (1998)

[13] M.L. Roukes, M.R. Freeman, R.S. Germain, R.C. Richardson, and M.B. Ketchen, Phys. Rev. Lett. 55, 422 (1985).

[IH] H. Hoevers, hitp://hwwisron.nLdivisions/srt/ 